\title{
Germanica
}

\section{Performing Lampedusa - Über europäische Grenz- und Migrationspolitik in Elfriede Jelineks Die Schutzbefohlenen, Hans-Werner Kroesingers FRONTex security und BBMs Po.W.E.R.}

Performing Lampedusa - On European frontier and migration politics in Elfriede Jelinek's Die Schutzbefohlenen, Hans-Werner Kroesinger's FRONTex security and BBM's Po.W.E.R.

Performing Lampedusa - Sur la politique européenne des frontières et de migration dans Die Schutzbefohlenen d'Elfriede Jelinek, FRONTex security de Hans-Werner Kroesinger et Po.W.E.R. de BBM.

\section{Simon Hagemann}

\section{OpenEdition}

Journals

Édition électronique

URL : http://journals.openedition.org/germanica/2912

DOI : 10.4000/germanica.2912

ISSN : 2107-0784

Éditeur

Université de Lille

Édition imprimée

Date de publication : 30 septembre 2015

Pagination : 125-140

ISBN : 9782913857353

ISSN : 0984-2632

\section{Référence électronique}

Simon Hagemann, « Performing Lampedusa - Über europäische Grenz- und Migrationspolitik in Elfriede Jelineks Die Schutzbefohlenen, Hans-Werner Kroesingers FRONTex security und BBMs

Po.W.E.R. », Germanica [Online], 56 | 2015, Online erschienen am: 30 September 2017, abgerufen am 06 Oktober 2020. URL : http://journals.openedition.org/germanica/2912 ; DOI : https://doi.org/10.4000/ germanica.2912 


\title{
Performing Lampedusa - Über europäische Grenz- und Migrationspolitik in Elfriede Jelineks Die Schutzbefohlenen, Hans-Werner Kroesingers FRONTex security und BBMs Po.W.E.R.
}

\author{
Simon HAGEMANN \\ Université de Franche-Comté
}

Abseits der größtenteils inhaltlich eher uninteressanten politischen Debatten um meistens faktenferne rechtspopulistische Forderungen nach einer größeren Steuerung von Einwanderung und einer stärkeren Begrenzung des Asylrechts, gerät die europäische Grenzpolitik in den letzten Jahren zumeist nach menschlichen Katastrophen in den Fokus der Öffentlichkeit. Im Zentrum der Aufmerksamkeit sind hierbei die spanischen Exklaven Ceuta und Melilla mit ihren Sicherheitszäunen und vor allem die immer wieder auftretenden Havarien überfüllter Flüchtlingsschiffe im Mittelmeer auf dem Weg von Afrika nach Europa. Ein trauriger Höhepunkt war hierbei die Schiffskatastrophe bei Lampedusa am 3. Oktober 2013 mit mindestens 366 ertrunkenen Passagieren. Dabei handelt es sich aber keinesfalls um Einzelfälle, 
sondern um einen Dauerzustand. Jeden Tag machen sich Menschen unter widrigsten Umständen auf die Reise vom afrikanischen zum europäischen Kontinent. Vorsichtige Schätzungen gehen von 3000 bis 4000 im Mittelmeer Ertrunkenen allein im Jahr 2014 aus ${ }^{1}$. Die alltäglichen menschlichen Tragödien werfen viele Fragen besonders in Bezug auf die strukturelle europäische Grenz- und Migrationspolitik auf. Darüber hinaus kann die Diskussion über eine europäische Identität und ein Europabild kaum ohne eine Berücksichtigung der Vorgänge an den europäischen Außengrenzen sowie der Flüchtlingssituation im Inneren geführt werden. Grenz- und Migrationspolitik sind auch wichtige Faktoren bei der Bestimmung eines europäischen Selbstverständnisses und einer europäischen Ethik.

Auch Künstler schalten sich zunehmend in diese wichtige gesellschaftliche Debatte ein. In den Jahren 2013 und 2014 sind drei Arbeiten von Protagonisten der deutschsprachigen Theater- und Performanceszene erschienen, die sich explizit mit der europäisierten Grenz- und Migrationspolitik beschäftigen: Elfriede Jelineks Theatertext Die Schutzbefohlenen (2013, Urlesung durch SchauspielerInnen und Asylbewerber in Hamburg, Uraufführung 2014 in Mannheim) ${ }^{2}$, die Dokumentartheaterarbeit von Hans-Werner Kroesinger FRONTex security (2013, Text und Inszenierung von Kroesinger) ${ }^{3}$, sowie das Stück Po.W.E.R. von BBM (Beobachter der Bediener von Maschinen) (2014, Text von Olaf Arndt, Inszenierung von BBM) ${ }^{4}$. Elfriede Jelinek, Literaturnobelpreisträgerin von 2004, ist bekannt für ihre postdramatischen Theatertexte, in denen Stimmen und Gegenstimmen für eine wortspielreiche Polyphonie sorgen und dabei oft ihren ideologischen Hintergrund offenlegen. Hans-Werner Kroesinger gilt wie kaum ein anderer Theatermacher im deutschsprachigen Raum als Vertreter des klassischen Dokumentartheaters. Seit vielen Jahren greift Kroesinger aktuelle Themen auf und bringt diese nach längerer Recherchearbeit auf die Bühne. Die Gruppe BBM ist seit Jahren eine feste Größe in der deutschsprachigen Medienkunstlandschaft und eine der wenigen Maschinenperformancegruppen in Deutschland5. Die Frage nach

1. - International Organisation for Migration (IOM) (herausgegeben von Tara Brian und Frank Laczko), Fatal Journeys. Tracking Lives Lost during Migration, International Organisation for Migration, Genf, 2014, S. 11.

2. - Unveröffentlicht, konsultierbar unter http://www.elfriedejelinek.com/ (letzter Zugriff 13.11.2014).

3. - Unveröffentlicht, Probenfassung.

4. - Druckfassung, Veröffentlichung geplant für Anfang 2015.

5. - Keineswegs ist dies als vollständig zu verstehen. So könnte als eine weitere Theaterarbeit zum Thema Migration die „Asyl-Monologe“ der Bühne für Menschenrechte um Michael Ruf genannt werden, welche die Lebenswege von Asylsuchenden und ihre Begegnung mit dem deutschen Asylsystem nachzeichnen. Natürlich setzen sich auch 
der Art und Weise, wie diese drei Theatertexte und die zugehörigen Inszenierungen auf die Vorgänge an den europäischen Außengrenzen blicken, lädt zu einer näheren Betrachtung ein. Es gilt zu untersuchen, welche politischen und sozialen Diskurse sie aufnehmen und auf die Probe stellen, auf welche ästhetischen Funktionsweisen sie zurückgreifen und welcher Erkenntnis- und Erfahrungsgewinn sich damit verbindet.

\section{Die Europäisierung der Grenz- und Migrationspolitik im Lichte von drei verschiedenen Theaterarbeiten}

Die aktuelle europäische Grenz- und Migrationspolitik ist das Resultat eines historischen Prozesses. Der entscheidende Schritt für die Europäisierung der Grenz- und Außenpolitik waren die Schengener Abkommen von 1985 (Schengen I) und 1990/1995 (Schengen II). Hier wurden nicht nur die Binnengrenzen der Europäischen Gemeinschaft/ Union abgebaut, sondern auch die ,europäische Außengrenze erfunden"6. Die 2004 gegründete Agentur Frontex ist der Versuch einer gemeinsamen europäischen Grenzpolitik an den EU Außengrenzen. Es handelt sich bei dieser Institution mit Sitz in Warschau um eine Koordination von Einsatzmitteln der Einzelstaaten. Die Agentur geriet des Öfteren in Verruf, da Vorwürfe laut wurden, Frontex hätte Flüchtlingsboote im Mittelmeer abgedrängt und bei unterlassener Hilfeleistung und unter Androhung von Gewalt Flüchtlinge ohne geordnetes Asylverfahren abgeschoben. Eine der letzten Entwicklungen der europäischen Grenzsicherung ist das 2013 beschlossene, sogenannte „European Border Surveillance System“, kurz EUROSUR genannt. Hierbei handelt es sich um ein Programm zum Informationsaustausch und um ein Überwachungssystem, welches neueste Technologien wie Drohnen, bewaffnete Roboter, Satellitensuchsysteme oder Aufklärungsgeräte einsetzen soll. Ziel ist es, die illegale Einwanderung nach Europa weiter zu erschweren. Das im Oktober 2013 von der italienischen Regierung gestartete Seenotrettungsprogramm Mare Nostrum wurde im Oktober 2014 durch die europäische Operation Triton ersetzt. Dabei verschiebt sich der Fokus immer mehr von der Flüchtlingsrettung zur Einreiseverhinderung.

Künstler in anderen Teilen der Welt mit der Grenz- und Migrationspolitik in ihren jeweiligen Regionen auseinander. Hier sei Version 1.0's CMI (A Certain Maritime Incident) (2004) über die Situation in Australien beispielhaft genannt.

6. - Sabine Hess, Vassilis Tsianos, „Europeanizing Transnationalism! Provincializing Europe! - Konturen eines neuen Grenzregimes“, in Transit Migration Forschungsgruppe (Hg.), Turbulente Ränder. Neue Perspektiven auf Migration an den Grenzen Europas, Bielefeld, Transcript, 2007, S. 28. 
Trotz aller europäischen Abschottung erfasst das oft zitierte statische Modell einer „Festung Europa“ gleich aus mehreren Gründen die Komplexität der Grenzpolitik und Migration an den europäischen Außengrenzen nicht. Das Verhältnis zwischen Europa und den MigrantInnen lässt sich kaum als ein einfacher zweipoliger Vorgang beschreiben. Wie Sabine Hess und Vassilis Tsianos zeigen, müssen viele verschiedene lokale und nationale Entscheidungsträger in die Überlegungen mit einbezogen werden?. Verschiedene Migrationsstrategien und (nationale wie europäische) politische Entscheidungen beeinflussen den Prozess. Dabei können die Interessen der verschiedenen Mitgliedsstaaten nicht als einheitlich betrachtet werden. Immer wieder kommt es zu Streitigkeiten zum Beispiel in Bezug auf Asylquoten zwischen den Mitgliedsstaaten. Die von Deutschland durchgesetzte Rechtskonstruktion „Sichere Herkunftsländer“ und die Drittstaatenregelung spielen hierbei eine Rolle. Weiterhin bedeutet die Europäisierung der Außenpolitik einen Prozess der Ausdehnung der europäischen Grenz- und Migrationspolitik, auch teilweise über die Grenzen Europas hinaus, wie beispielsweise die Debatte über die Errichtung von EU-Auffanglagern in Afrika zeigt. Außerdem kann die EU durch den Erweiterungsprozess der Mitgliedstaaten ihre Grenzkompetenzen räumlich ausdehnen. Migration nach Europa und die europäische Grenz- und Migrationspolitik ist somit nur als ein dynamischer Prozess unter Berücksichtigung vielerlei Faktoren zu verstehen.

Die drei Theaterarbeiten von Jelinek, Kroesinger und Arndt versuchen sich dieser Komplexität anzunähern. Auch wenn bei der Untersuchung, wie sie dies tun, die Texte im Vordergrund stehen sollen, so werden auch Elemente der verschiedenen zugehörigen Inszenierungen in die Überlegungen mit einbezogen. Vor allem bei den Arbeiten von Kroesinger und BBM sind Text und Aufführung schwer zu trennen, da die Autoren auch die Regisseure sind, und somit das Denken an die szenische Umsetzung bereits in den Schreibprozess, zumindest teilweise, mit eingeflossen sein dürfte.

Dokumentartheater, wie es von Peter Weiss definiert wurde, ist eine Theaterform, die auf verschiedenen Dokumenten (zum Beispiel Zitate, Akten, Zeitungsartikel, Fotos, Filme, Briefe, Statistiken oder Interviews) basiert ${ }^{8}$. Es benutzt authentisches Material und adaptiert dieses für die Bühne. Die Auswahl und die Formgebung transformieren

7. - Ebd., S. 24-25.

8. - Peter Weiss, „Notes sur le théâtre documentaire“ (1967), in Discours sur la genèse et le déroulement de la très longue guerre de libération du Viêt Nam illustrant la nécessité de la lutte armée des opprimés contre leurs oppresseurs ainsi que la volonté des États-Unis d'Amérique d'anéantir les fondements de la révolution, Paris, Éd. du Seuil, 1968, S. 7-15. 
dabei das Material in ein künstlerisches Werk. Hans-Werner Kroesinger bleibt dem Ansatz von Weiss im Wesentlichen verpflichtet. Dem Stück ging eine längere Recherchephase mit der Dramaturgin Regine Dura voraus. So enthält das Stück eine große Menge an Informationen über die Geschichte und die aktuellen Entwicklungen der europäischen Grenzsicherung und des europäischen Asylrechts. Das Material wird einerseits von den vier Sprechern abwechselnd vorgetragen, andererseits finden eine Reihe von Interviews mit Protagonisten der europäischen Grenz- und Asylpolitik statt. Zu Wort kommen Frontex Direktor Ilkka Laitinen, der Vorsitzende des Verwaltungsrates von Frontex Ralf Göbel, EU-Innenkommissarin Cecilia Malström, der ehemalige deutsche Innenminister Otto Schily und die Bürgermeisterin von Lampedusa Giusi Nicolini. Außerdem findet sich im Text ein Ausschnitt einer Verhandlung vor dem Europäischen Gerichtshof für Menschenrechte. Das reichhaltige Material wird dabei in kleinen Abschnitten gesampelt. Es wird nie direkt kommentiert. Ein Kommentar ergibt sich nur durch die Anordnung und Konfrontation der Materialien beziehungsweise durch die Bühnenumsetzung. Kroesinger hat selbst als Regisseur seinen Text auf die Bühne gebracht (Uraufführung im HAU Berlin). Der teilweise trockene und komplizierte Text im Beamtenjargon wird von vier SchauspielerInnen in schlichter Bürokleidung lebendig vorgetragen. Dabei werden die Zuschauer mit Informationen geradezu überfrachtet. Oft, gerade bei den Interviews ist aber eine feine Ironie zu spüren, welche eine gewisse Distanz zum Gesagten schafft. Ein Souffleur sorgt dafür, dass gelegentliche Aussetzer schnell übersprungen werden und wirkt gleichzeitig illusionshemmend. Für einige kontrastvolle Pausen sorgt eine Opernsängerin im glitzernden Kleid mit ihrem Gesang über Schuld und Opfer. Die weißen Wände und die Büroeinrichtung auf der Bühne vermitteln eine kalte Behördenatmosphäre. An den Rändern der Bühne werden auf Monitoren die verschiedenen Abkürzungen von EU-Projekten und deren Erklärungen eingeblendet. Die „Eurokratie“ ist so immer präsent. Nach circa zwei Dritteln des Stücks fragt ein Schauspieler, was passieren würde, wenn die Grenzen offen wären. Daraufhin werden die Zuschauer aufgefordert, auf die Hinterseite der Bühne zu kommen. Dort sitzen diese nun U-förmig um eine kleine Bühne angeordnet. In diesem zweiten Teil geht es nun konkreter um die Vorkommnisse auf Lampedusa. Vor allem wird die unterlassene Hilfeleistung der Behörden beim Schiffsunglück thematisiert.

Po.W.E.R. Poseidon Welfare Enhancement and Rescue Service von BBM erschien in der Reihe Die Welt ohne uns (2010-2014) des Staatstheaters Hannover. Die Arbeiten dieser Aufführungsreihe beschäftigen sich mit einer radikalen Zukunftsvision in der die Erde sich ohne menschliches Leben weiterentwickelt. Po.W.E.R. war der letzte von insgesamt neun Beiträgen. Obwohl Po.W.E.R. sich in diese 
Serie einschreibt, geht es inhaltlich um die Vorgänge an den europäischen Außengrenzen im Jahr 2014. Der dramaturgische Trick, der die Brücke von der Zukunft in die Gegenwart schlägt: Die Zuschauer begeben sich auf eine Reise in die Zukunft ohne menschliches Leben, von der aus dann Maschinen auf die Zeit um 2014 herum zurückblicken. Wie FRONTex security enthält der Text eine Reihe von Informationen zur europäischen Grenzüberwachung. Die informative Reise führt über verschiedene Stationen bis ins Herz der Zentrale von Frontex in Warschau, den sogenannten Warroom oder Situation Room. Hier, 10000 Jahre in der Zukunft, diskutieren mehrere Roboter und versuchen herauszufinden, was Menschenrechte, vom Klima ausgelöste Migration, Grenzen oder Wachstum am Anfang des 21. Jahrhunderts bedeuteten. Der Text enthält eine Reihe ironischer Kommentare. Außerdem gibt es immer wieder musikalische Gesangseinlagen. Aufgeführt wurde das Stück im teilweise stillgelegten Kraftwerk Herrenhausen in Hannover. Die in schwarzen Sicherheitsanzügen uniformierten Schauspieler führen die Zuschauer durch die besondere Kulisse bis hin in den Frontex Warroom, der mit riesigen Pixelwänden der Medienkunstgruppe BlinkenArea ausgestattet ist. Die Bildschirmwände symbolisieren die Überwachungseinheiten, von denen aus die Frontexmitarbeiter die Grenzvorgänge überwachen. In der Mitte des Raumes bewegen sich die selbststeuernden Maschinen, welche (gesprochen von menschlichen Sprechern) über die Vergangenheit diskutieren.

Wie auch in den meisten anderen von Jelineks Theatertexten ist der Text in Die Schutzbefohlenen keinen bestimmten Sprechern zugeordnet und besteht nur aus mehreren großen Textblöcken, enthält eine Vielzahl von Sprachspielen und Anspielungen und viele unmarkierte Zitate. Deren wichtigste Quellen nennt Jelinek im Paratext selbst: Die Schutzflehenden von Aischylos, Die Metamorphosen von Ovid, eine „Prise Heidegger" und eine Broschüre des österreichischen Staatssekretariats für Integration des Bundesministeriums für Inneres. Das Asyl- und Menschenrechtsthema, der bittere, klagende Sprachstil sowie der Titel können auf den Aischylostext bezogen werden. Aus den aktiv Flehenden werden aber die passiv Befohlenen, was wiederum eine höhere Verantwortung für die Gastgeber impliziert. Die realen Ereignisse, welche am meisten im Text Erwähnung finden, sind zum einen die Asylproteste in der Wiener Votivkirche und zum anderen die Flüchtlingssituation auf Lampedusa. Der Theatertext ist überwiegend von einer Stimme aus MigrantInnensicht gesprochen, was vor allem in dem Dualismus „Wir und Ihr“ deutlich wird. Trotz der MigrantInnenposition liegt der Fokus weniger auf den Lebenswegen der MigrantInnen, denn auf der aufnehmenden europäischen Gesellschaft. Außerdem haben sich in die Ausführungen dieser Stimme darüber hinaus auch andere Diskurse, beziehungsweise Gegenstimmen, eingeni- 
stet. Im Gegensatz zu Kroesinger und BBM ist Elfriede Jelinek nicht für die Inszenierung ihres Textes verantwortlich. Die Uraufführung übernahm der erfahrene Jelinekregisseur Nicolas Stemann. Der Inszenierung voraus ging aber eine Urlesung im Oktober 2013 in der Hamburger St. Pauli Kirche. Dabei traten neben den Thalia Theater SchauspielerInnen auch Flüchtlinge der Lampedusa Gruppe auf ${ }^{9}$. Das Stück wurde dann im Mai 2014 im Rahmen des Festivals "Theater der Welt” in Mannheim uraufgeführt.

Die drei Arbeiten unterscheiden sich somit sowohl in der Perspektive als auch in der ästhetischen Herangehensweise. Aber sie stellen ähnliche Fragen. Der Blick auf die MigrantInnen, ihre Motivationen und die sich herausbildenden Migrationsnetzwerke stehen dabei nicht im Mittelpunkt. Es geht vor allem um die aktuelle Situation der europäischen Grenz- und Migrationspolitik. Besonders wichtig scheinen dabei die Inszenierung der europäischen Grenzpolitik und Grenzüberwachung und die Bestimmung einer europäischen Identität, welche sich im Spannungsverhältnis zwischen Menschenrechtsdiskurs auf der einen und der Migrationsrealpolitik auf der anderen Seite verorten lässt.

\section{Die Inszenierung der Grenzpolitik und Grenzüberwachung}

Wie lassen sich die komplexen Vorgänge an den europäischen Außengrenzen künstlerisch erfahrbar machen? Hans-Werner Kroesinger nähert sich einer Systemdarstellung durch historische Kontextualisierung, beispielsweise mit Exkursen zum Asylbegriff der Antike, aber vor allem zu den Momenten, an denen sich die europäische Asylpraxis verändert hat, andererseits vertieft er die Vorgänge mit Fachwissen wie beispielsweise dem sehr eindringlich geschilderten Ertrinken aus medizinischer Sicht. Des Weiteren verfolgt Kroesinger manchmal eine Strategie des Reenactements, wenn Interviews mit den Protagonisten oder der Prozess vor dem europäischen Menschenrechtsgerichtshof wiedergegeben werden. Durch die präzise Wiedergabe des Beamtenjargons, welcher über Menschenleben entscheidet, werden die politischen Mechanismen offengelegt. Dabei wirkt in der Aufführung der Pragmatismus der Interviewten leicht überzeichnet und die Schauspieler wahren eine gewisse ironische Distanz zu ihren Rollen. Auffällig in der Aufführung ist außerdem das Zurückgreifen auf Miniaturmodelle. Insgesamt gibt es nur wenige Bühnenaccessoires. Ganz am Anfang werden gelbe Sterne auf eine blaue Europafahne geklebt. Später werden Tassen mit Aufdrucken von

9. - Eine Gruppe von ca. 300 Bürgerkriegsflüchtlingen aus Libyen, die seit Anfang 2013 für ein dauerhaftes Bleiberecht kämpft. 
EU-Länderflaggen herumgeschoben. Im zweiten Teil bewegen und versenken die SchauspielerInnen Miniaturboote. Mit dem wiederholten Einsetzten von Miniaturmodellen thematisiert sich das dokumentarische Theater auch selbst und fragt nach den Möglichkeiten von Mimesis und Repräsentation. Mit den ästhetischen Strategien der Montage von diversem Recherchematerial, beispielsweise der Konfrontation der EU-Bürovorgänge mit den Geschehnissen im Mittelmeer, der Kontextualisierung, dem ironisch-distanzierten Reenactement und der Miniatisierung versucht Kroesinger den vielschichtigen und historisch gewachsenen Frontex-Komplex für die Zuschauer erfahrbar zu machen und gleichzeitig die Möglichkeiten der Annährung an die Problematik zu thematisieren.

Bei Po.W.E.R. verweist ebenfalls bereits der Titel auf die Thematik der europäischen Grenzpolitik, denn dieser steht für Poseidon Welfare Enhancement and Rescue service, wobei „Poseidon“ das Codewort für das Frontexeinsatzgebiet im östlichen Mittelmeer ist. Das Stück von BBM nähert sich thematisch vor allem zwei Aspekten der Grenzpolitikproblematik, dem sicherheitstechnischen und dem wirtschaftlichen. Es beginnt mit einer Lautsprecheransage einer DPA Meldung über die Forderung von Nichtregierungsorganisationen nach einem Verbot autonomer Waffen. Hauptaugenmerk gilt dem TalosProgramm, bei dem zur europäischen Grenzsicherung halb-automatische Systeme eingesetzt werden sollen ${ }^{10}$. Ziel sind ,intelligente Grenzen"11, mit teilweise automatisierten Überwachungsprogrammen, welche potenziell mit nicht-letalen Waffen, vor allem dem TASER ausgerüstet werden können. Po.W.E.R. ist in diesem Zusammenhang auch eine Fortsetzung von Olaf Arndts vorherigen Studien zu Sicherheits- und Überwachungsdispositiven und nicht-letalen Waffen ${ }^{12}$. Frontex spielt bei dem Erproben neuer Technologien und dem Einsatz von vorgeblich humanitären Waffensystemen eine Schlüsselrolle ${ }^{13}$. Der zweite Schwerpunkt der Arbeit von BBM liegt auf der Betonung des ökonomischen Aspektes bei der Grenz- und Migrationspolitik. Die gesamte Vielfalt einer „Migrationsindustrie“ wird in Po.W.E.R. ausführ-

10. - Siehe http://www.talos-border.eu/.

11. - O. Arndt, Po.W.E.R., a.a.O., S. 12.

12. - Olaf Arndt, Demonen, Zur Mythologie der Inneren Sicherheit, Hamburg, Edition Nautilus, 2005.

13. - Hierzu Olaf Arndt im Programmheft von Po.W.E.R. (S.31-32): ,Zweitens soll Frontex die Forschungsprojekte für die neuen Technologien bestimmen, also gemeinsam mit den Einsatzkräften vor Ort ermitteln, welche Technologien für den Einsatz vor Ort notwendig sind. Und dafür nehmen sie dann natürlich hauptsächlich Technologien, die sich schon als neue humanitäre Techniken erwiesen haben, so wie der Taser, der zwar unerträgliche Schmerzen auslöst und dich lähmt, aber nicht tötet.“ 
lich seziert ${ }^{14}$. Zum einen geht es um die Sicherheitsindustrie und ihre Verdienstmöglichkeiten an den EU-Programmen zur Grenzsicherung. Firmen wie der Hersteller von tracking devices Thales oder der Rüstungskonzern Finmecanica, welcher unter anderem Drohnen und Roboter herstellt, werden konkret benannt. Die Migrationsindustrie wird umfassender auch auf Firmen wie die Lufthansa bezogen, welche an Abschiebungen mitverdienen. Ein gewisser Migrationsstrom hat somit für eine Reihe von Unternehmen einen ganz praktischen ökonomischen Nutzen. Außerdem wird ein gewisser Zuzug von MigrantInnen als durchaus gewollt bezeichnet, da illegale MigrantInnen vor allem für Arbeiten im Niedrigsektor notwendig seien. So heißt es in Po.W.E.R., dass es daher oft keine klaren Entscheidungen zum Rechtsstatus der Migranten gebe ${ }^{15}$. Auch für die Betreiber von Auffanglagern und Nichtregierungsorganisationen (NGOs) ist wie Heidrun Friese im Programmheft von Po.W.E.R. ausführt, die Aufnahme von mobilen Menschen

zu einem einträglichen Geschäft geworden. Allein das Jahr 2013 brachte den (privaten) Betreibern der italienischen Aufnahmelager 1,8 Mill. Euro ein und auch an der Aufnahme von unbegleiteten Minderjährigen kann verdient werden: Die Betreiber der Einrichtungen bekommen 70 Euro/Tag. Auch NGOs haben Anteil an dem Geschäft und konkurrieren auf dem Spendenmarkt. [...] Auch Terre des Hommes engagierte sich und hat im Jahr 2011 ein Projekt auf der Insel begonnen. 2013 gelang Terre des Hommes auch die Zusammenarbeit mit $C \& A$. Im Winter spendete die Modefirma $1 \%$ ihrer Einnahmen in Italien dem Projekt. Auch durften die Kunden ihren Beitrag leisten und beim Kleiderkauf an der Kasse spenden. Der >Notfall< Lampedusa wandert an die Kassen, verbindet sich mit multinationalen Unternehmen, deren sozialer Imagepflege. Konsum wird zu Opferkonsum mit verkäuflicher Moral. ${ }^{16}$

Die Verknüpfung der Migration mit der globalen Ökonomie erfolgt in Po.W.E.R. auch über zwei der Roboter im Frontexhauptquartier mit den Namen Goldmann und Sax, welche unter anderem über die Automatisierung im Finanzwesen philosophieren ${ }^{17}$ und darüber hinaus den Zusammenhang zwischen Ökonomie und Sicherheitstechnik symbolisieren. Die selbstgesteuerten Maschinen, die riesigen Bildschirme, die uniformierten Soldaten und die Kulisse des Kraftwerkes sorgen dafür, dass die Sicherheitstechnik für die Zuschauer auch physisch erfahrbar

14. - O. Arndt, Po.W.E.R., a.a.O., S. 15.

15. - Ebd., S. 15.

16. - Heidrun Friese, "Programmheft zu Po.W.E.R.", Niedersächsiches Saatstheater Hannover GmbH, Schauspiel Hannover, 2014, S. 41-42.

17. - O. Arndt, Po.W.E.R., a.a.O., S. 59. 
gemacht wird. Dazu wird das gesamte Stück mit Augenzwinkern als ein „Reality Game“ bezeichnet ${ }^{18}$, also ein Spiel unter Einbeziehung von Wirklichkeitselementen, wobei sich die Zuschauer mit Helmen und festen Schuhen vor den Gefahren der Wirklichkeit schützen können.

Elfriede Jelineks Theatertext Die Schutzbefohlenen hat einen etwas anderen Fokus als die Texte von Arndt und Kroesinger. Hier steht zunächst nicht die EU-Grenzpolitik, sondern die Situation der MigrantInnen in Österreich und in Deutschland im Vordergrund. Vor allem die Situation in und um die Wiener Votivkirche und den Hungerstreik der Männer aus Pakistan, Afghanistan und Nordafrika. In der zweiten Hälfte des Stückes finden aber auch die unerträglichen Vorgänge vor und auf Lampedusa Erwähnung, wenn es heißt ,so viele von uns werden nie ankommen“. Am Ende des Textes auf der Homepage von Elfriede Jelinek ist ein Bild eines Flüchtlingsbootes auf dem Meer zu sehen. Im starken Kontrast zu den Vorgängen im Mittelmeer steht die immer wieder im Text erwähnte österreichische Blitzeinbürgerung der Tochter des ehemaligen russischen Präsidenten Boris Jelzin. Der ökonomische und machtpolitische Faktor der Grenz- und Einbürgerungspolitik wird so von Jelinek mehr als deutlich gemacht.

\section{Die Thematisierung europäischer Identität zwischen Menschenrechtsdiskurs und Migrationsrealpolitik}

Aus den Überlegungen zur Darstellung der europäischen Grenzpolitik, ergibt sich die Frage, wie darüber hinaus allgemein die europäische Migrations- und Asylpolitik und die sich daraus ergebenden Konsequenzen für die europäische Identität in den Theatertexten erläutert werden. Das Textgewebe der Schutzbefohlenen wird von Stimmen und Gegenstimmen durchzogen. Jelinek selbst hat den Begriff der Sprachflächen ins Spiel gebracht. Es handelt sich dabei nicht um eine Imitation der menschlichen Alltagssprache. Vielmehr werden verschiedene Diskurse aufeinander bezogen. Somit lässt sich der Text sowohl aufgrund seiner Heterogenität als auch aufgrund seiner sprachlichen Beschaffenheit nicht auf eine Sprecherrolle im Sinne der Mimesis reduzieren, auch wenn die Anklage der Migrantensituation dominiert und teilweise mit konkreten Familien- und Lebensbezügen unterstrichen wird. Doch ihre schwierigen Familienbiografien finden in der Aufnahmegesellschaft keinerlei Berücksichtigung: „Auf die Toten können wir uns ja nicht berufen, wenn wir ein Aufenthaltsrecht ableiten wollen, die wollen uns hier ja selber ableiten wie Flüsse, ins Nirgendwo.“ Das vorherige Leben der MigrantInnen spielt keine Rolle mehr, es gilt, sich den Weisungen der Werbetexte der Hochglanzbroschüre

18. - Ebd., S. 3. 
„Zusammenleben in Österreich“ unterzuordnen. Bei der Montage der Broschüretexte und den Situationsbeschreibungen der MigrantInnen offenbart sich eine starke Diskrepanz. Die Stimme beklagt das Verschwinden der Individualität, bei der die Asylsuchenden „keine einzelnen Menschen mehr sind“", beziehungsweise nicht als solche wahrgenommen werden. Auch dadurch, dass innerhalb des Wir-Diskurses aus MigrantInnensicht die anderen Diskurse eingebettet sind, wird die selbstbestimmte Rede der MigrantInnen bereits auf sprachlicher Ebene in Frage gestellt.

„Welche Grundlage außer der Menschenwürde bleibt uns übrig?“", fragt die Stimme. Eine Berufung auf den Menschenrechtsdiskurs scheint die letzte Option. Der Menschenrechtsdiskurs hat für Europa eine fundamentale Bedeutung und, wie Bärbel Lücke ausführt, intoniert Jelineks Chor der Schutzbefohlenen "nicht nur das Klagen und litaneihafte Flehen, er beruft sich sachkundig (eine überlagerte AutorinstanzStimme ist da zu hören) auf die Menschenrechte, deren Wurzeln durchaus ebenso in der Antike zu verorten sind wie das Problem von Flucht und Asyl"19. Doch auch das Berufen auf das Menschenrecht scheint keinen großen Effekt zu haben. Denn die ,über Katzenvideos Gerührten" scheinen keine Notiz von den MigrantInnen zu nehmen. Diese klagen „Wir sind gekommen, doch wir sind gar nicht da“. Der Menschenrechtsdiskurs droht sich in der konkreten Situation zu verflüchtigen und so heißt es: „Machen sie ein Foto von der Menschenwürde bevor sie wieder weg ist". Überhaupt wird über den gesamten Text immer wieder das Fotografieren und Videoerstellen erwähnt. Doch eine Aussagekraft scheinen die erstellten Dokumente kaum mehr zu haben, sondern verkommen zu Erzeugnissen leerer Schaulust und nichtssagenden Zeitvertreibs. Neben dem Menschenrechtsdiskurs bildet Heideggers Dasein-Begriff (vor allem aus Sein und Zeit und Kant und das Problem der Metaphysik) den philosophischen Bezugspunkt auf den sich die Migrantenstimme mehrmals bezieht, um ihre ausweglos erscheinende Situation zu beschreiben. Bärbel Lücke führt hierzu aus:

Dass die Schutzflehenden sich jeweils als Dasein verstehen, dem die Existenzgrundlage entzogen ist - wobei Jelinek häufig zwischen der humanistischen oder der Alltags-Terminologie (Existenz im allgemeinen Verständnis) und der Heideggerschen Sprache wechselt, z. B. wenn es heißt: „die Menschenwürde [...] ergibt sich durch unsere Existenz als Menschen, und wenn wir keine Menschen sind, haben wir auch keine Würde" -, ist daraus ersichtlich, dass sie (heideggerianisch) fordern: „Wir wollen sein“. Denn es werden ihnen alle

19. - Bärbel Lücke, ,Aischylos, Aufklärung und Asylproteste in Österreich (und anderswo). Zu Elfriede Jelineks Stück Die Schutzbefohlenen“, in Textem. Texte und Rezensionen, http://www.textem.de/index.php?id=2519 (letzter Zugriff 29.11.2014). 
„Seinsmöglichkeiten des Miteinanderseins“ verweigert, ebenso „die weite Mannigfaltigkeit vertretbarer Weisen des In-der-Welt-Seins“".20

Das Stück endet im Scheitern:

Dass uns Recht geschieht, darum beten wir, das erfülle mein Gebet um freies Geleit, um ein Los, das gewinnt, um ein besseres Los, aber es wird nicht geschehen. Es wird nicht geschehen. Es ist nicht. Wir sind gar nicht da. Wir sind gekommen, doch wir sind gar nicht da. ${ }^{21}$

Mit der Situationsbeschreibung der Schutzbefohlenen, denen selbst ein Berufen auf die elementarsten Menschenrechte nicht hilft, zeichnet Jelinek ein Bild von Europa, welches sich weit von seinen eigenen Ansprüchen entfernt hat.

Als Wertekoordinaten im Europa von Die Schutzbefohlenen scheinen nur noch Legalität, Geld und Macht zu zählen. So wird die Versessenheit der Europäer auf die Legalität beklagt, für die „legal“ zu einem „Lieblingswort“ geworden ist. Außerdem wird der enorme politische Einfluss des Großkapitals in der Person des Multimillionärs und Politikers Frank Stronach denunziert. Durch das Verweben mit dem Europamythos aus Ovids Metamorphosen stellt Jelinek einen direkten Bezug zur europäischen Identität her. Jelinek verknüpft den Mythos von Zeus als verwandeltem Stier und Europa mit der internationalen Finanzindustrie. Passend ist dabei, dass der Stier auch als Symbol für steigende Börsenkurse gilt. Bärbel Lücke erläutert den Zusammenhang von Europa und Finanzindustrie in Die Schutzbefohlenen:

Verschmolzen mit dem Mythos der Nymphe Io, die von Zeus in eine weiße Kuh verwandelt wurde, ist Europa (,Europa, nein io, Ich, der südliche Mensch [...], von einem Gott geschwängert") selbst die Kuh, und die Götter sind die mächtigen Konzernherren, die, die geschwängerte Kuh weidend (,Demokratie, ihre Hufe, die hinweggleiten über uns"), das Weideland Demokratie zertreten. ${ }^{22}$

InFRONTexsecurity fälltauf, dass die Stimmen derFlüchtlinge fehlen. Die Vertreter der europäischen Sicherheitspolitik dominieren. Selbst in der Verhandlungsszene am europäischen Menschenrechtsgerichtshof in Strassburg sprechen nicht die Flüchtlinge, sondern die sie vertretenden Anwälte ${ }^{23}$. In dem Rechtsstreit zwischen einer Gruppe von Flüchtlingen und dem italienischen Staat über eine Abschiebung auf hoher See

20. - Ebd.

21. - E. Jelinek, a.a.O.

22. - B. Lücke, a.a.O.

23. - H.-W. Kroesinger, a.a.O., S. 19. 
entspricht das Gericht der Anklage der Flüchtlinge ${ }^{24}$. Doch an der Praxis ändert dies wenig. Es wird deutlich, dass Grenzsicherungspolitik und Menschenrechtsanspruch in einem Spannungsverhältnis stehen. FRONTex security untersucht, was die Menschenrechte in einer technokratischen Welt noch wert sind. Das ,it works“ von Frontexchef Laitinen als Antwort auf die Frage nach der Beurteilung der Frontexarbeit scheint sich immer mehr als Leitmotiv durchzusetzen ${ }^{25}$. Dabei kommen in FRONTex security auch Äußerungen von Verantwortlichen des europäischen Grenzschutzes vor, welche menschliche Sorgen in Bezug auf die humanitäre Situation im Mittelmeerraum äußern ${ }^{26}$. Allerdings werden diese Aussagen durch die Montagetechnik mit der Praxis der Grenzüberwachung und der Abschiebepraxis konfrontiert und verlieren daher an Glaubwürdigkeit. Immer mehr wird im Laufe des Stückes das europäische Selbstverständnis hinterfragt. Dies wird von Hans-Werner Kroesinger auch in einem Interview mit Peter Laudenbach explizit benannt:

Der Name dieser Agentur ist schon sehr theatralisch: Frontex. Eine Front bedeutet, dass man sich gegen Feinde verteidigen und sie angreifen muss. Heißt das, dass aus Sicht der Europäischen Union Afrikaner, die in Europa Asyl suchen, unsere Feinde sind? [...] Armut hat politische Ursachen. Weshalb ist die Flucht vor lebensbedrohender Armut oder aus Bürgerkriegsländern aus Sicht der EU ,illegal“? Man hat auf der einen Seite die Selbstbilder eines demokratischen, aufgeklärten, toleranten, sozialen Europas. Gleichzeitig entwickelt dieses Europa, politisch gewollt und gut organisiert, eine große Härte und Brutalität gegenüber Menschen, deren einziges Verbrechen darin besteht, dass sie leben wollen wie wir. Dieser Kontrast zwischen offiziellem Selbstbild und realem Handeln ist theatralisch ergiebig. Letztlich ist das Stück auch eine Selbstkritik. ${ }^{27}$

Aber auch das Stück selbst ist sehr direkt in seiner europäischen Selbstkritik, wenn die Opernsängerin von mit Blut befleckten Händen singt oder es heißt, durch die „Betonung der Illegalität mit Frontex hat die EU das gesamte Mittelmeer zu einem Kampfgebiet erklärt" 28 . Die Frage nach der humanitären Identität der EU wird so, ähnlich wie

24. - Ebd., S. 21.

25. - Ebd., S. 3.

26. - Ebd., S. 11.

27. - Peter Laudenbach, „Hans-Werner Kroesinger über sein Stück FRONTex security “, Interview in Tipp Berlin, vom 12.12.2013, verfügbar unter http://www.tip-berlin. de/kultur-und-freizeit-theater-und-buehne/hans-werner-kroesinger-uber-sein-stuck-frontex-security (zuletzt konsultiert am 15.11.2014).

28. - H.-W. Kroesinger, a.a.O., S. 7. 
bei Jelinek, allerdings mit anderem Blickwinkel, unmissverständlich gestellt.

Auch bei der Arbeit von BBM stellt sich diese Frage. Po.W.E.R. thematisiert die europäische Identität vor allem mit Blick auf den Einsatz von Sicherheitstechnologien und deren ökonomische Grundlagen und gesellschaftliche Auswirkungen (Schengen Schengen freies Land Doch dann kommt die Hohe Mauer Grenze geht durch Körper dann $\left.{ }^{29}\right)$. Schon in früheren Arbeiten hat sich Olaf Arndt ausgiebig mit Sicherheitstechnologien beschäftigt und er schreibt: „An diesen Waffen nämlich lässt sich ablesen, wie der Mensch sich verändert. An ihnen können wir sehen, wie wir uns unsere Zukunft und letztlich uns selbst vorstellen." ${ }^{30}$ Dass der Einsatz von nichtletalen Waffen bei der Grenzsicherung nicht wirkungslos für die europäische Identität sein wird, benennt Po.W.E.R. ebenso, wie es die Verschleierung und diskursive Uminterpretation von den Vorgängen an den europäischen Grenzen kritisiert. So heißt es, dass die europäischen Grenschutzprogramme gerade deswegen griechische Namen tragen, da dann niemand sich fragen würde, ob sie im Widerspruch zur europäischen Kultur stehen würden ${ }^{31}$. Weiterhin wird erklärt, dass Klimaflüchtlinge gerne als Wirtschaftsflüchtlinge deklariert werden, um eine europäische Verantwortung herunterzuspielen ${ }^{32}$. Außerdem wird die Umetikettierung von Grenzkontrollmaßnahmen als Rettungsmaßnahmen kritisiert ${ }^{33}$. $\mathrm{Zu}$ den diskursiven Problemen kommen aber auch ganz praktische Überlegungen, so wie wenn es um Fischer geht, die administrative Probleme bekommen, wenn sie MigrantInnen im Meer auflesen und helfen ${ }^{34}$. Wie die Grenzvorgänge auf das europäische Selbstverständnis einwirken, wird auch ausdrücklich formuliert. So heißt es: „Das ist die Stärke unserer Kultur: die totale Entkoppelung von Wissen und Gewissen."35 Die Frage der Verantwortung wird auch von den sich selbst steuerenden Maschinen thematisiert, wenn sie fragen: „Sollen wir für das Ausführen von Befehlen schuldig gesprochen werden?"36 Der besondere Gag am Ende von Po.W.E.R. ist die Erklärung im Gespräch der Maschinen für das Aussterben der Menschen. Bei genmanipulierten Maisbroten wurde ein Abgleich mit den bürgerlichen Tugenden in den Code eingeschmuggelt. Der Abgleichung der bürgerlichen Tugenden

29. - O. Arndt, Po.W.E.R., a.a.O., S.4.

30. - O. Arndt, Demonen, Zur Mythologie der Inneren Sicherheit, a.a.O., S. 18.

31. - O. Arndt, Po.W.E.R., a.a.O., S. 56.

32. - Ebd., S. 15.

33. - Ebd., S. 46.

34. - Ebd., S. 8.

35. - Ebd., S. 8.

36. - Ebd., S. 53. 
mit dem realen Verhalten der rassistischen Filterung von Einwanderung und der unsozialen Finanzpolitik hielten die Menschen so nicht stand und schafften sich konsequenterweise selber $\mathrm{ab}^{37}$. Unmissverständlich kritisiert Po.W.E.R. so den Werteverlust der bürgerlichen europäischen Gesellschaft und das völlige Primat von Nationalismus und Ökonomie.

\section{Auf Europa und seine Grenzen blicken: Zugänge und Perspektiven}

Eine der wichtigsten Fragen des politischen Theaters ist die Frage nach den Zugängen zur Realität. Alle drei hier besprochenen Arbeiten zeichnen sich durch ausgiebige Recherchen aus und enthalten dokumentarische Elemente. Auch wenn die Zugänge von BBM und Jelinek wesentlich freier sind und sich nur Kroesingers Arbeit in die klassische Tradition des Dokumentartheaters einordnen lässt. Wie Alison Forsyth und Chris Megson bemerken, begreift das Dokumentartheater die historische Wahrheit als ein Feld der Auseinandersetzung und denkt dabei auch über seine eigenen Bedingungen nach ${ }^{38}$. Dies lässt sich auch allgemein auf politisches Theater übertragen. Die hier analysierten drei Theaterarbeiten thematisieren in diesem Sinne die verschiedenen Prozesse und Diskurse in Bezug auf die europäische Grenz- und Migrationspolitik. Dabei werden Strategien einer immanenten Subversion angewendet, da manche Diskurse sich in einem neuen Zusammenhang von selbst entlarven. Verstärkt wird dies durch die Montage des Materials, die starke Gegensätze zwischen Diskursen und Realitäten hervortreten lässt. Außerdem werden durch Kontextualisierung und Historisierung die aktuellen Vorgänge in einen größeren Zusammenhang gestellt. Auch die Frage der Möglichkeiten von Kunst, sich gesellschaftlichen Prozessen zu nähern, wird teilweise aufgeworfen. Das Dokumentarische befindet sich, wie Janelle Reinelt bemerkt, nicht ausschließlich im Objekt, sondern zwischen Objekt, Mediator (Künstler oder Historiker) und den Zuschauern ${ }^{39}$. Dies gilt auch für das Politische im Allgemeinen. So tragen die Arbeiten von Jelinek, Kroesinger und BBM nicht einfach nur Recherchematerial zusammen, sondern beziehen durch die Montage und Ironisierung mancher Passagen klar eine Position, und fordern so auch die Zuschauer zur Positionierung heraus. Es wird deutlich gemacht, dass Frontex auch im Namen aller EU-ZuschauerInnen handelt und Grenz- und

37. - Ebd., S. 68-69.

38. - Alison Forsyth, Chris Megson (Hg.), Get Real: Documentary Theatre Past and Present, London, Palgrave Macmillan, [2009], 2011, siehe "Introduction" von A. Forsyth, C. Megson, S. 3.

39. - Siehe ebd.: Janelle Reinelt, "The Promise of Documentary", S. 7. 
Migrationspolitik gesamtgesellschaftliche Aufgaben sind. Die drei Arbeiten zeigen so das kritische Potenzial von Theatertexten, europäische Migrations- und Grenzpolitik zu prüfen, politische, ökonomische und soziale Zusammenhänge aufzuzeigen, Widersprüche offen zu legen und vorherrschende Diskurse zu stören. Visionen oder Utopien für ein Europa der Menschenrechte scheinen in diesem Zusammenhang sehr schwierig, beziehungsweise sind sie an eine konkrete Handlung gebunden. Wie die Arbeiten von BBM, Kroesinger und Jelinek zeigen, können Utopien einer friedlichen, weltoffenen und freiheitlichen europäischen Identität nicht fernab der täglichen Grenz- und Migrationspolitik entwickelt werden, sondern müssen im Gegenteil ständig mit der Realpolitik abgeglichen werden. 